\title{
Sympathetic Cooling of Trapped Ions: A Laser-Cooled Two-Species Nonneutral Ion Plasma
}

\author{
D. J. Larson, ${ }^{(a)}$ J. C. Bergquist, J. J. Bollinger, Wayne M. Itano, and D. J. Wineland \\ National Bureau of Standards, Time and Frequency Division, Boulder, Colorado $80303^{\circ}$
}

(Received 31 March 1986)

\begin{abstract}
Sympathetic cooling of trapped ions has been demonstrated in an experiment where ${ }^{198} \mathrm{Hg}^{+}$ions were confined in a Penning ion trap with laser-cooled ${ }^{9} \mathrm{Be}^{+}$ions. ${ }^{198} \mathrm{Hg}^{+}$temperatures below $1 \mathrm{~K}$ were achieved. Ion plasma sizes, shapes, and rotation frequencies were measured. Dramatic changes in the ${ }^{9} \mathrm{Be}^{+}$plasma were observed when the ${ }^{198} \mathrm{Hg}^{+}$ions were introduced into the trap. These observations are consistent with the prediction of centrifugal separation for ions of different charge-to-mass ratios.
\end{abstract}

PACS numbers: $32.80 . \mathrm{Pj}, 52.25 . \mathrm{Wz}$

The techniques of trapping and laser cooling have led to dramatic improvements in recent measurements and future prospects for precision spectroscopy. ${ }^{1,2}$ However, the number of species which can be directly laser cooled is limited by the availability of tunable, narrow-band sources and by more fundamental problems such as complicated level structure (as in molecules) or the absence of optical structure (as in protons and negative atomic ions). In addition, the laser-cooling process itself strongly perturbs the levels of an atom or ion. The cooling laser must be turned off during the interrogation time to avoid light shifts (ac Stark shifts) and broadening many orders of magnitude larger than achievable linewidths. In the most accurate experiment to date, ${ }^{3}$ the linewidth of a radio frequency transition in ${ }^{9} \mathrm{Be}^{+}$was limited $(\geqslant 0.025$ $\mathrm{Hz}$ ) by the necessity to restrict interrogation times $(\leqslant 20 \mathrm{~s})$ in order to minimize heating that occurred when the cooling laser was turned off. In principle, linewidths substantially less than $0.001 \mathrm{~Hz}$ should be possible on transitions in trapped ions.

Sympathetic cooling, ${ }^{1,4,5}$ where one species is cooled by interaction with a second, directly cooled species, offers a solution to these problems. In the present experiments, spectroscopy of continuously cooled ${ }^{198} \mathrm{Hg}^{+}$ions was made possible by confining them in a trap together with laser-cooled ${ }^{9} \mathrm{Be}^{+}$ions. The $313-\mathrm{nm}$ radiation used to $\mathrm{cool}$ the $\mathrm{Be}^{+}$ions did not seriously perturb the $\mathrm{Hg}^{+}$ions since this wavelength is far from any resonance in $\mathrm{Hg}^{+}$. We estimate that the fractional light shift in the ${ }^{201} \mathrm{Hg}^{+}$hyperfine separation would be less than $5 \times 10^{-18}$ under conditions similar to those in the present experiment.

Although sympathetic cooling was noted previously in experiments with different isotopes of magnesium ions ${ }^{1,4}$ we report here the first measurements of temperatures and spatial distributions for a two species, magnetically confined, nonneutral plasma. In addition, the present experiments have demonstrated that radial diffusion of the sympathetically cooled species is prevented. One interesting feature of such a plasma, that the species with higher mass-to-charge ratio moves radially to the outside of the lighter (or more highly charged) species, ${ }^{6,7}$ was observed. The present results appear to have a direct bearing on proposed experiments to store and cool antiprotons at very low energies $^{8-10}$ and may be important in other experiments where two or more species of different charge-to-mass ratio are simultaneously present in the trap. ${ }^{11}$ Different considerations lead to separation of species in if traps. $^{12}$

The experiments were carried out using a Penning ion $\operatorname{trap}^{13}$ with hyperbolically shaped electrodes. The endcap-to-endcap spacing was $0.51 \mathrm{~cm}$ and the innerring diameter was $0.83 \mathrm{~cm}$. The trap and vacuum system were at room temperature for all of the measurements except one where the trap was cooled to $77 \mathrm{~K}$. A magnetic field near $1.43 \mathrm{~T}$ was used for all of the measurements. $\mathrm{Be}^{+}$-ion plasmas (or "clouds") were created in the trap by use of a beam of electrons to ionize beryllium atoms which were evaporated from a tungsten filament wrapped with beryllium wire. $\mathrm{Hg}^{+}$ ions were loaded into the trap by ionizing mercury vapor leaked into the vacuum system through a variable leak valve while the $\mathrm{Be}^{+}$ions were being laser cooled.

Three laser systems were used to cool and probe the ions. A frequency-doubled dye laser beam with a power of approximately $50 \mu \mathrm{W}$ at $313 \mathrm{~nm}$ was directed through a hole in the ring electrode and used to cool the $\mathrm{Be}^{+}$ions on the $2 s^{2} S_{1 / 2}$ to $2 p^{2} P_{3 / 2}, m_{J}=+\frac{1}{2}$ to $m_{J}=+\frac{3}{2}$, transition. ${ }^{1}$ The photons backscattered by the $\mathrm{Be}^{+}$ions were collected and counted. This signal was used for all of the measurements on the $\mathrm{Be}^{+}$ions. A second 313-nm source was directed through another hole in the ring. When tuned to the $2 s^{2} S_{1 / 2}$ to $2 p^{2} P_{3 / 2}, m_{J}=+\frac{1}{2}$ to $m_{J}=-\frac{1}{2}$, transition, this probe beam depopulated the $m_{J}=+\frac{1}{2}$ state and thus caused a decrease in the scattered light from the cooling beam. ${ }^{1}$ The ion-cloud sizes and shapes were measured by observing this signal for different positions of the probe beam. The ion-cloud rotation frequencies were obtained through the Doppler shift by measuring the frequency of the probe laser which produced maximum depopulation. ${ }^{14}$ A third laser system ${ }^{15}$ produced 
a few microwatts of tunable power at $194 \mathrm{~nm}$. The positions and velocities of the $\mathrm{Hg}^{+}$ions in the trap were measured by collecting and counting the $194-\mathrm{nm}$ photons scattered when the frequency was tuned through the $6 s^{2} S_{1 / 2}$ to $6 p^{2} P_{1 / 2}, m_{J}=-\frac{1}{2}$ to $m_{J}=+\frac{1}{2}$ transition in the ion. Microwaves tuned to the $\Delta m_{J}=1$ transition in the ground state prevented depletion of the $m_{J}=-\frac{1}{2}$ ground state by optical pumping. ${ }^{16}$ For both $\mathrm{Be}^{+}$and $\mathrm{Hg}^{+}$the temperatures were determined by measuring the Doppler contribution to the frequency width of the observed signal as the appropriate laser was tuned through resonance. ${ }^{14}$ An example of the signal from a $\mathrm{Hg}^{+}$-ion cloud whose cyclotron temperature has been sympathetically cooled to near $1 \mathrm{~K}$ is shown in Fig. 1.

A cold, single-species ion cloud in a Penning trap with hyperbolic electrodes should assume a nearly spheroidal shape with constant density inside the cloud and an abrupt drop in density at the edge. ${ }^{14,17,18}$ The eccentricity of the spheroid is determined by the trapping voltage, magnetic field, and rotation frequency. Any cloud in thermal equilibrium will exhibit uniform rotation. The rotation frequency is a measure of the density and of the angular momentum of the ions. ${ }^{17-21}$ It can be partially controlled by varying the torque applied to the cloud by the cooling laser. Observed rotation frequencies were well below those corresponding to the maximum stable density of ions in the trap, the Brillouin density. ${ }^{20}$ The mechanism which limits the frequency is not clearly understood, but it may depend upon trap asymmetries. ${ }^{18,19,22}$ For precision spectroscopy, high rotation velocities and the accompanying Doppler shifts should be avoided by working at low densities.

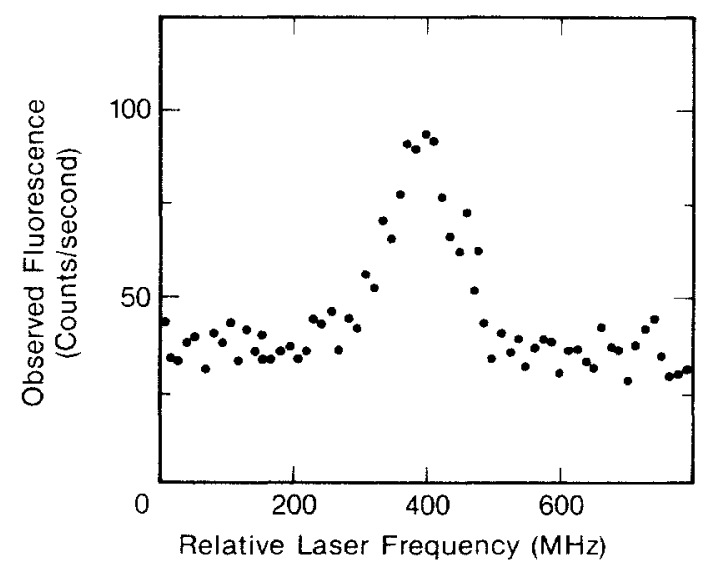

FIG. 1. Observed fluorescence from a $\mathrm{Hg}^{+}$-ion cloud cooled to approximately $1 \mathrm{~K}$ by interaction with a lasercooled $\mathrm{Be}^{+}$cloud. The horizontal axis is the frequency of a laser tuned through the $6 s^{2} S_{1 / 2}\left(m_{J}=-\frac{1}{2}\right)$ to $6 p^{2} P_{1 / 2}$ $\left(m_{J}=+\frac{1}{2}\right)$ transition in $\mathrm{Hg}^{+}$. The room-temperature Doppler width of this transition is about $1.4 \mathrm{GHz}$.
A dramatic change in the rotation frequency (and thus the density) and the shape (away from spheroidal) of the $\mathrm{Be}^{+}$cloud occurred when $\mathrm{Hg}^{+}$ions were introduced into the trap. With only $\mathrm{Be}^{+}$in the trap, rotation frequencies up to nearly $200 \mathrm{kHz}$ could be achieved. The single-particle rotation (magnetron) frequency was measured to be only $9.4 \mathrm{kHz}$ with -1.5 $\mathrm{V}$ on the ring, so the observed frequencies were due primarily to the space-charge fields. With $\mathrm{Hg}^{+}$and $\mathrm{Be}^{+}$in the trap, the largest observed rotation frequency was $21 \mathrm{kHz}$. The measured size and shape of a $\mathrm{Be}^{+}$ cloud with and without $\mathrm{Hg}^{+}$is shown in Fig. 2(a). Adding the $\mathrm{Hg}^{+}$ions decreased the rotation frequency by a factor of 2. A larger change in density and shape of a $\mathrm{Be}^{+}$cloud is shown in Fig. 2(b). Figures 2(a) and 2(b) are the smallest and largest clouds examined. In all cases the $\mathrm{Be}^{+}$clouds were prolate spheroids without the $\mathrm{Hg}^{+}$and oblate and nonspheroidal with $\mathrm{Hg}^{+}$present. The $\mathrm{Hg}^{+}$clouds appeared disk shaped with diameter-to-length ratios of 6 to 1 or larger.

Clearly, the $\mathrm{Hg}^{+}$ions applied a substantial torque to the $\mathrm{Be}^{+}$ions. For all of the $\mathrm{Hg}^{+}$clouds measured, the axial bounce frequency (equal to twice the axial length divided by the rms axial velocity) was approximately equal to the rotation frequency. This condition is consistent with axial asymmetries of the trap and resultant resonant particle transport limiting the rotation frequency. ${ }^{18,22}$ No definitive evidence is available, however, to exclude other mechanisms ${ }^{23}$ from limiting the rotation frequency with the exception of drag due to background gas. Cooling of the trap electrodes to $77 \mathrm{~K}$, which substantially lowered the residual mercury pressure, caused no change in measured rotation frequencies. Despite the presence of a torque on the $\mathrm{Hg}^{+}$ions, they remained in the trap for several hours unless the cooling was interrupted. If the cooling laser was blocked or tuned off resonance the $\mathrm{Hg}^{+}$ ions were lost in several minutes, demonstrating that $\mathrm{Hg}^{+}$confinement was dependent on the torque applied to the $\mathrm{Hg}^{+}$ions by the laser via the coupling to the $\mathrm{Be}^{+}$ions.

Measured $\mathrm{Hg}^{+}$temperatues ranged from 0.4 to 1.8 $\mathrm{K}$ and $\mathrm{Be}^{+}$temperatures ranged from less than 0.05 to $0.2 \mathrm{~K}$. The temperature difference between the two species may be a result of the thermal coupling between the directly laser-cooled $\mathrm{Be}^{+}$ions and the indirectly cooled $\mathrm{Hg}^{+}$ions being weak, due to centrifugal separation. Also, the axial temperature of the $\mathrm{Be}^{+}$ ions should be higher than the transverse temperature because of photon recoil, ${ }^{14}$ and this could cause the $\mathrm{Hg}^{+}$-ion temperature to take on some intermediate value.

Given the torque due to the $\mathrm{Hg}^{+}$ions, the counteracting torque due to the laser on the $\mathrm{Be}^{+}$ions, and the temperature difference between the two clouds, it is not unreasonable to expect some shear. In a uni- 


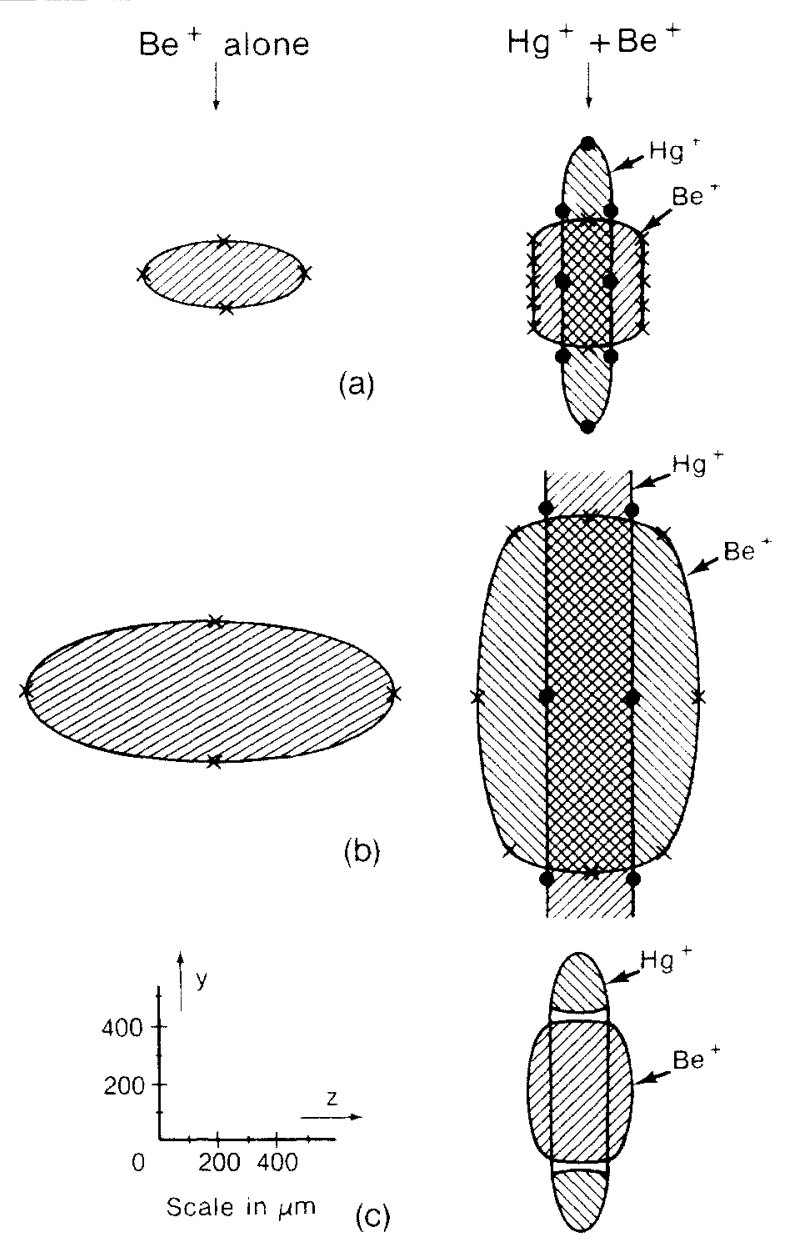

FIG. 2. (a) Measured $\mathrm{Be}^{+}-$and $\mathrm{Hg}^{+}$-ion-cloud shapes. The magnetic field is along the $z$ axis. The $\mathrm{Be}^{+}$cloud, which contained about 800 ions, is shown with and without the $\mathrm{Hg}^{+}$present. The measured rotation frequencies were 41 $\mathrm{kHz}$ without $\mathrm{Hg}^{+}$[corresponding to a density, $\left.n\left(\mathrm{Be}^{+}\right)=3.9 \times 10^{7} \mathrm{~cm}^{-3}\right\}$ and $20 \mathrm{kHz}$ with $\mathrm{Hg}^{+}$present $\left[n\left(\mathrm{Be}^{+}\right)=1.9 \times 10^{7} \mathrm{~cm}^{-3}, n\left(\mathrm{Hg}^{+}\right)=1.6 \times 10^{7} \mathrm{~cm}^{-3}\right]$. The solid lines drawn through the data points conform to the theoretically predicted and separately confirmed spheroidal shape for $\mathrm{Be}^{+}$alone but are drawn without consideration of theoretical shapes for $\mathrm{Be}^{+}$and $\mathrm{Hg}^{+}$together. The edges of the clouds were measured with an uncertainty of about 25 $\mu \mathrm{m}$. (b) Measured cloud shapes for a $\mathrm{Be}^{+}$cloud which contained about 12000 ions and a $\mathrm{Hg}^{+}$cloud which was larger in radial extent than the aperture through the ring electrode (diameter $=2100 \mu \mathrm{m}$ ). The measured rotation frequencies were $73 \mathrm{kHz}$ without $\mathrm{Hg}^{+}\left[n\left(\mathrm{Be}^{+}\right)=7.0 \times 10^{7} \mathrm{~cm}^{-3}\right]$ and $18 \mathrm{kHz}$ with $\mathrm{Hg}^{+}\left[n\left(\mathrm{Be}^{+}\right)=1.8 \times 10^{7} \mathrm{~cm}^{-3}, n\left(\mathrm{Hg}^{+}\right)\right.$ $=1.5 \times 10^{7} \mathrm{~cm}^{-3} \mathrm{l}$. (c) Theoretically predicted shapes for cold $\mathrm{Be}^{+}$and $\mathrm{Hg}^{+}$clouds under conditions similar to those in (a).

formly rotating cloud, the Doppler shift and thus the observed optical resonance frequency should be a linear function of the radial distance from the center of the cloud. Figure 3 shows the observed resonance frequency for $\mathrm{a} \mathrm{Be}^{+}$cloud, confined in the trap together

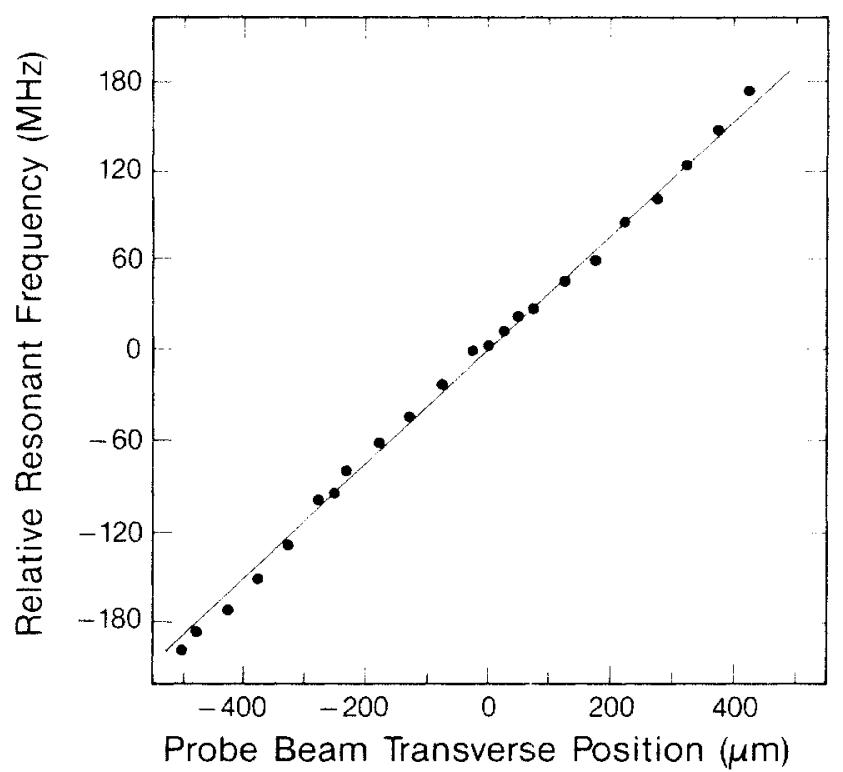

FIG. 3. Measured resonance frequency in $\mathrm{Be}^{+}$as a function of the transverse position (perpendicular to the trap axis) of the probe laser beam. These measurements, done with $\mathrm{Hg}^{+}$ions present in the trap, placed a limit on the amount of shear in the $\mathrm{Be}^{+}$cloud. The statistical error in the data points is smaller than the size of the points.

with a $\mathrm{Hg}^{+}$cloud, as a function of the transverse position of the probe laser beam. Fitting the data in Fig. 3 with a rotation frequency $\omega=\omega_{r}+\Delta \omega\left(r / r_{\mathrm{cl}}\right)$, where $r_{\mathrm{cl}}$ is the cloud radius, implies that $\left|\Delta \omega / \omega_{r}\right|<0.3$. Indeed, to the extent that the data suggest that shear is present, it appears that the outer edge of the cloud is rotating faster than the middle. No evidence for shear was observed in a similar measurement on $\mathrm{Hg}^{+}$ions or in the comparison of the frequencies between the two species.

The measured shapes of the $\mathrm{Be}^{+}$- and $\mathrm{Hg}^{+}$-ion clouds were compared to numerical calculations based on a zero-temperature theory which balances magnetic, space-charge, and external electric forces. ${ }^{6,17,24}$ The calculation used measured values for the trapping voltage, magnetic field, rotation frequency, and relative cloud diameters to predict shapes. A zerotemperature theory should be applicable when the ratio of the Debye length to the dimensions of the ion cloud is small. In the present experiments this ratio was typically less than 0.1 . The theoretical prediction of complete radial separation of the ion clouds was not definitively tested since all of the probing of ion position was done in a direction perpendicular to the axis of the trap. Changes in collection efficiency for the 194-nm fluorescence as the beam position was moved prevented the use of count rate as a measure of the $\mathrm{Hg}^{+}$cloud thickness along the laser-beam direction. The theory ${ }^{6,24}$ suggests that the heavier $\mathrm{Hg}^{+}$ions should be found in a torus around the lighter $\mathrm{Be}^{+}$ions 
as shown in Fig. 2(c).

These experiments have demonstrated the effectiveness of sympathetic cooling and point the way toward applications such as use in high-resolution spectroscopy and antiproton accumulation and storage. They have also allowed observation of some of the characteristics of a cold, magnetically confined, two-species nonneutral plasma. Significant features include the dramatic change in the shape of the $\mathrm{Be}^{+}$-ion cloud when the $\mathrm{Hg}^{+}$ions are introduced into the trap, the temperature difference between the laser-cooled $\mathrm{Be}^{+}$ ions and the sympathetically cooled $\mathrm{Hg}^{+}$ions, and the substantial torque exerted on the $\mathrm{Be}^{+}$cloud by the $\mathrm{Hg}^{+}$ions. A few features, including the source of the torque on the $\mathrm{Hg}^{+}$ions, are not clearly understood and may limit the number of ions that can be stored in a trap. Such techniques may be applicable to cooling neutral particles in a trap. ${ }^{25}$

We would like to thank J. Prestage, R. Hulet, and C. Manney for their contributions to this work. We thank T. O'Neil, C. F. Driscoll, L. Brewer, and F. Walls for helpful comments on the manuscript. We gratefully acknowledge the support of the U.S. Office of Naval Research and of the U.S. Air Force Office of Scientific Research.

${ }^{(a)}$ Permanent address: Department of Physics, University of Virginia, Charlottesville, VA 22901.

'For reviews, see D. J. Wineland, W. M. Itano, J. C. Bergquist, J. J. Bollinger, and J. D. Prestage, in Atomic Physics 9, edited by R. S. Van Dyck, Jr., and E. N. Fortson (World Scientific, Singapore, 1985), p. 3; W. D. Phillips, J. V. Prodan, and H. Metcalf, ibid., p. 338, and references therein.

${ }^{2}$ The Mechanical Effects of Light, edited by P. Meystre and S. Stenholm, J. Opt. Soc. Am. B 2, No. 11 (1985).

3J. J. Bollinger, J. D. Prestage, W. M. Itano, and D. J. Wineland, Phys. Rev. Lett. 54, 1000 (1985).

${ }^{4}$ R. E. Drullinger, D. J. Wineland, and J. C. Bergquist, Appl. Phys. 22, 365 (1980).

${ }^{5}$ D. J. Wineland, R. E. Drullinger, and F. L. Walls, Phys.
Rev. Lett. 40, 1639 (1978).

6T. M. O'Neil, Phys. Fluids 24, 1447 (1981).

${ }^{7}$ M. Geva, M. Krishnan, and J. L. Hirshfield, J. Appl. Phys. 56, 1398 (1984).

${ }^{8}$ H. G. Dehmelt et al., Bull. Am. Phys. Soc. 24, 757 (1979); G. Torelli, in Proceedings of the Fifth European Symposium on Nucleon Anti-Nucleon Interactions, Bressanone, Italy, 23-28 June 1980, edited by M. Cresti (Istituto Nazionale di Fisica Nucleare, Padua, Italy, 1980), p. 43.

${ }^{9} \mathrm{G}$. Gabrielse, H. Kalinowsky, and W. Kells, in Physics with Antiprotons at LEAR in the ACOL Era, edited by U. Gastaldi et al. (Editions Frontières, Gif-sur-Yvette, France, 1985), p. 665; W. Kells, IEEE Trans. Nucl. Sci. 32, 1770 (1985)

${ }^{10} \mathrm{C}$. F. Driscoll, in Proceedings of the Anti-Matter Facility Workshop (Univ. of Wisconsin Press, Madison, 1985); M. H. Holzscheiter, ibid.

${ }^{11}$ D. A. Church, in Ref. 1, p. 137.

${ }^{12}$ C. Flory, L. Cutler, and R. Giffard, to be published.

${ }^{13}$ H. G. Dehmelt, Adv. At. Mol. Phys. 3, 53 (1967), and 5, 109 (1969); D. J. Wineland, W. M. Itano, and R. S. Van Dyck, Jr., Adv. At. Mol. Phys. 19, 135 (1983).

$14 \mathrm{~J}$. J. Bollinger and D. J. Wineland, Phys. Rev. Lett. 53, 348 (1984).

${ }^{15}$ H. Hemmati, J. C. Bergquist, and W. M. Itano, Opt. Lett. 8, 73 (1983).

16W. M. Itano, J. C. Bergquist, and D. J. Wineland, J. Opt. Soc. Am. B 2, 1392 (1985).

${ }^{17}$ S. A. Prasad and T. M. O'Neil, Phys. Fluids 22, 278 (1979)

${ }^{18 D}$ D. J. Wineland, J. J. Bollinger, W. M. Itano, and J. D. Prestage, J. Opt. Soc. Am. B 2, 1721 (1985)

${ }^{19}$ J. H. Malmberg, C. F. Driscoll, and W. D. White, Phys. Scr. T 2, 288 (1982).

${ }^{20}$ R. C. Davidson, Theory of Nonneutral Plasmas (Benjamin, Reading, MA, 1974), p. 4.

21T. M. O'Neil and C. F. Driscoll, Phys. Fluids 22, 266 (1979).

${ }^{22}$ C. F. Driscoll and J. H. Malmberg, Phys. Rev. Lett. 50, 167 (1983).

23D. L. Eggleston, T. M. O'Neil, and J. H. Malmberg, Phys. Rev. Lett. 53, 982 (1984).

${ }^{24} \mathrm{~J}$. J. Bollinger, C. Manney, and D. J. Wineland, to be published.

25W. H. Wing, Prog. Quantum Electron. 8, 181 (1984). 